\title{
Clopidogrel did not inhibit platelet function early after coronary bypass surgery: A prospective randomized trial
}

\author{
Eric Lim, MRCS \\ Jacqueline Cornelissen, MPhil \\ Tom Routledge, MRCS ${ }^{a}$ \\ Stephen Kirtland, $\mathrm{PhD}^{\mathrm{a}}$ \\ Susan C. Charman, $\mathrm{MSc}^{\mathrm{b}}$ \\ Sarah Bellm, BSc ${ }^{a}$ \\ Helen Munday, RGN ${ }^{\mathrm{a}}$ \\ Omar Khan, MRCS ${ }^{a}$ \\ Imran Masood, MRCS ${ }^{a}$ \\ Stephen Large, FRCS ${ }^{\mathrm{a}}$
}

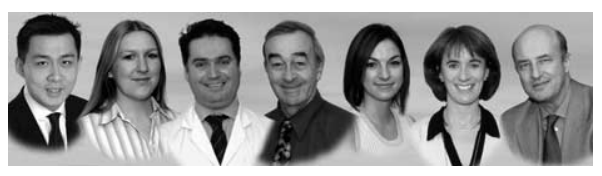

Lim, Cornelissen, Routledge, Kirtland, Bellm, Munday, Large (left to right)
From the Departments of Cardiothoracic Surgery and Clinical Pharmacology, Papworth Hospital, ${ }^{\mathrm{a}}$ and the Medical Research Council Biostatistics Unit, ${ }^{\mathrm{b}}$ Cambridge, United Kingdom.

Supported by Papworth Hospital NHS Trust and the Papworth Hospital Surgeons Research Fund. E.L. is currently supported by the Medical Research Council, United Kingdom

Presented at the Annual Meeting of the Society of Cardiothoracic Surgeons of Great Britain and Ireland, Guernsey, United Kingdom, March 7, 2004.

Received for publication Jan 25, 2004; revisions received Feb 19, 2004; accepted for publication March 4, 2004.

Address for reprints: Eric Lim, Department of Cardiothoracic Surgery, Papworth Hospital, Papworth Everard, Cambridge CB3 8RE, United Kingdom (E-mail: eric.lim@ cvsnet.org)

J Thorac Cardiovasc Surg 2004;128:432-5 $0022-5223 / \$ 30.00$

Copyright $\odot 2004$ by The American Association for Thoracic Surgery

doi:10.1016/j.jtcvs.2004.03.007
Objective: Although the beneficial effect of aspirin prescription after coronary surgery has been established, the efficacy of clopidogrel has never been compared with that of aspirin in the critical early postoperative period. We therefore conducted a prospective, double-blind, randomized controlled trial to compare the efficacies of these antiplatelet regimens.
Methods: Patients undergoing elective primary coronary artery bypass surgery were invited to participate. After the operation, patients were randomized to receive 100 $\mathrm{mg}$ aspirin, $325 \mathrm{mg}$ aspirin, or $75 \mathrm{mg}$ clopidogrel tablets daily for 5 days. Our primary outcome measure was platelet aggregation on day 5, expressed as percentage of baseline. Assessment of platelet aggregation was undertaken with the technique of Born.

Results: From September 2002 to July 2003, a total of 54 patients were randomized into the study. There were 2 self-withdrawals and 2 protocol violations, leaving 50 patients for analysis, 34 in the aspirin group and 16 in the clopidogrel arm. Compared with baseline, the mean percentage aggregations with collagen on day 5 were $56 \%$ for aspirin and $99 \%$ for clopidogrel. The mean difference between the two arms was $42 \%$ (95\% confidence interval 27\%-56\%) in favor of aspirin. At the same time point, the effective concentration to inhibit 50\% aggregation in the samples from patients randomly assigned to receive clopidogrel were not raised for our entire panel of agonists (changes of $-0.04 \mu \mathrm{g} / \mathrm{L}$ for collagen, $-0.01 \mu \mathrm{mol} / \mathrm{L}$ for epinephrine, and $-0.02 \mu \mathrm{mol} / \mathrm{L}$ for adenosine diphosphate).

Conclusion: Clopidogrel, unlike aspirin, did not inhibit platelet aggregation in the first 5 postoperative days and therefore should not be used as a sole antiplatelet agent early after coronary surgery.

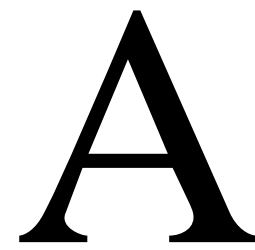

ntiplatelet therapy after coronary bypass is simple to administer, cost-effective, and critical. Although the beneficial effects of aspirin in the prevention of graft occlusion has been well established, controversies have emerged regarding the optimal aspirin $\operatorname{dose}^{1}$ and indeed the ideal antiplatelet agent. It has been reported that low-dose aspirin $(100 \mathrm{mg})$ does not effectively inhibit platelet function after coronary surgery, ${ }^{2}$ and a subanalysis from the CAPRIE study (Clopidogrel versus Aspirin in Patients at Risk of Ischemic Events) reported striking reductions in the risk of recurrent ischemic events for patients receiving clopidogrel 


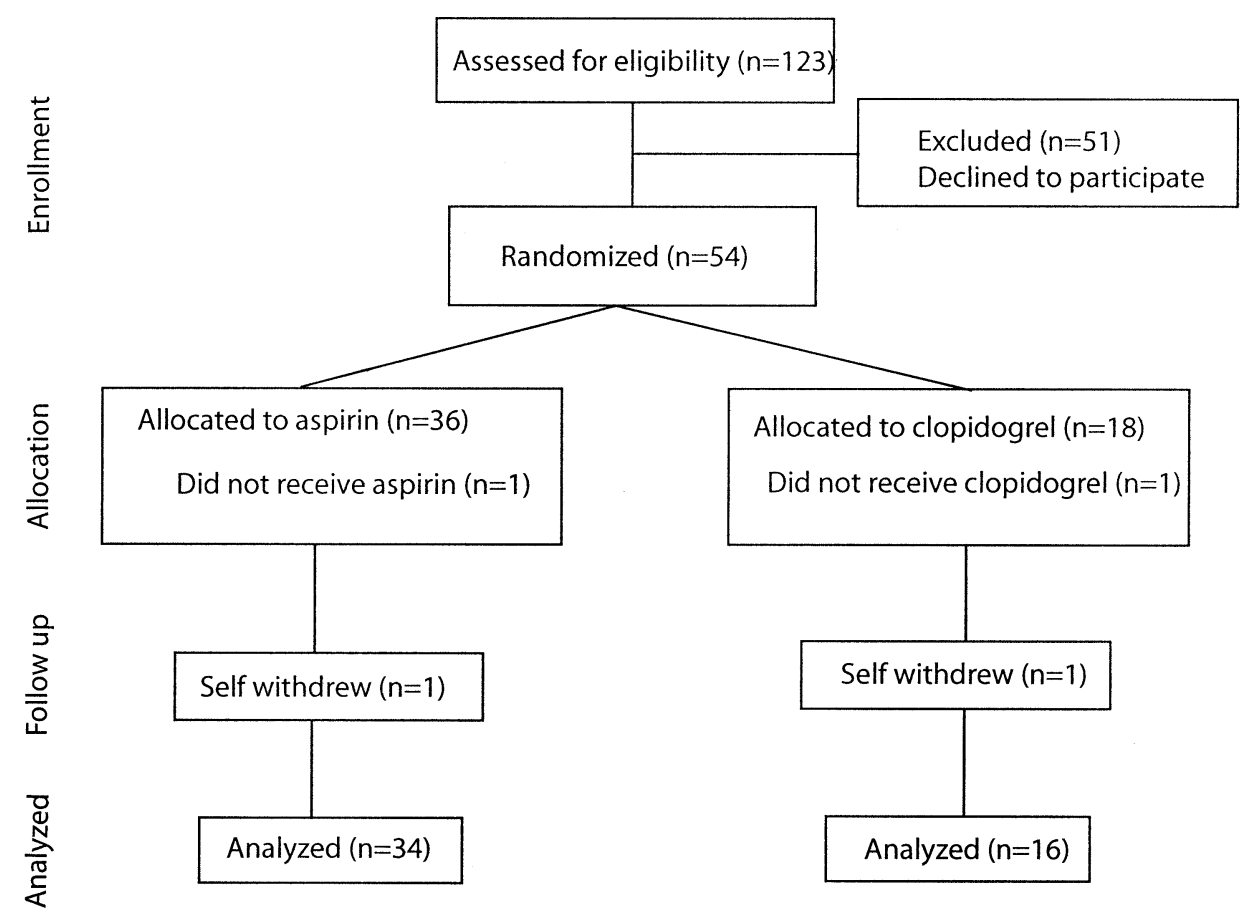

Figure 1. Trial flow diagram.

with previous history of cardiac surgery. ${ }^{3}$ This controversy led us to investigate the effects of low- and medium-dose aspirin and clopidogrel (as an alternative antiplatelet agent) on inhibition of platelet function after coronary artery bypass surgery.

\section{Methods}

We report here the results of an analysis based on interim data from a local research ethics committee-approved prospective, randomized trial currently in progress at our institution. We planned to recruit 108 patients (36 in each of the three arms), with an interim analysis planned after 54 patients (18 in each arm). We aimed to be able to detect a difference of $30 \%$ in postoperative platelet aggregation at day 5 (expressed as a percentage of preoperative values) between any two arms. If this difference was found to be statistically significant (at $2 \%$ level), the inferior arm would be stopped. At the analysis stage there would be at least $90 \%$ power to detect a difference of at least 1.5 SDs.

All patients undergoing elective primary coronary artery bypass surgery were invited to participate, and we excluded patients if they did not discontinue antiplatelet therapy a week before surgery, had contraindications to aspirin or clopidogrel, were receiving other medications that interact with aspirin or clopidogrel, underwent surgery without cardiopulmonary bypass, received platelet transfusion during the operation or within the first 24 hours, or could not be extubated in the first 24 hours.

No restrictions were placed on operative technique, and cardiopulmonary bypass was driven by a roller pump with a membrane oxygenator with an arterial (but no leukocyte) filter, as standard at our institution. Temperature on bypass ranged between $30^{\circ} \mathrm{C}$ to $37^{\circ} \mathrm{C}$. Tranexamic acid (but not aprotinin) was administered routinely.

On the first postoperative morning, patients were randomly assigned to receive one of the following identically encapsulated treatments: $100 \mathrm{mg}$ aspirin, $325 \mathrm{mg}$ aspirin, or $75 \mathrm{mg}$ clopidogrel daily for 5 days. Randomization was undertaken by the pharmacy into treatment allocation blocks of 6 .

Our primary outcome measure was percentage aggregation on day 5 ( 2 hours after drug administration) expressed as percentage of baseline, with $1.1 \mu \mathrm{g} / \mathrm{L}$ collagen as an agonist. Assessment of platelet aggregation was undertaken according to the technique of Born. ${ }^{4}$ Secondary outcome measures were effective concentrations of Horm collagen, adenosine diphosphate (ADP), and epinephrine on day 5 required to produce $50 \%$ aggregation $\left(\mathrm{EC}_{50}\right)$ relative to baseline.

For this analysis, patients were grouped according to treatment allocation of aspirin or clopidogrel. Categoric data are presented as frequency (percentage), and continuous data are given as mean \pm $\mathrm{SD}$ or median with interquartile range. Comparisons of categoric data between the two groups were made with the Fisher exact test or Pearson $\chi^{2}$ test. Continuous data were compared with the Student $t$ test or the Mann-Whitney test as appropriate.

\section{Results}

From September 2002 to July 2003, a total of 123 patients were invited to participate, 72 of whom consented to participate in the study. After enrollment, 54 patients were randomly allocated into study groups. Four patients were withdrawn, 2 as self-withdrawals and 2 because of protocol 
TABLE 1. Patient characteristics

\begin{tabular}{lccc}
\hline & Aspirin & Clopidogrel & $\boldsymbol{P}$ value \\
\hline Sample size & 36 & 18 & \\
Age (y, mean \pm SD) & $65 \pm 8$ & $64 \pm 8$ & .67 \\
Mean height, m (SD) & $1.7 \pm 0.1$ & $1.7 \pm 0.1$ & .28 \\
Mean weight, kg (SD) & $86 \pm 15$ & $82 \pm 11$ & .35 \\
Male sex (No.) & $35(97 \%)$ & $17(94 \%)$ & .61 \\
Diabetes (No.) & $6(17 \%)$ & $5(28 \%)$ & .34 \\
Hypercholesterolemia (No.) & $33(92 \%)$ & $16(89 \%)$ & .74 \\
Hypertension (No.) & $19(53 \%)$ & $8(44 \%)$ & .56 \\
Current smoker (No.) & $2(6 \%)$ & $3(17 \%)$ & .18 \\
No. of grafts (median, IQR) & $4(3-4)$ & $3(3-4)$ & .44 \\
Bypass (min, median, IOR) & $72(63-85)$ & $64(55-78)$ & .14 \\
Cholesterol lowering therapy (No.) & & & \\
$\quad$ None & $4(11 \%)$ & $1(6 \%)$ & \\
Atorvastatin & $12(33 \%)$ & $8(44 \%)$ & \\
Simvastatin & $10(28 \%)$ & $3(17 \%)$ & .70 \\
Pravastatin & $9(25 \%)$ & $6(33 \%)$ & \\
Fluvastatin & $1(3 \%)$ & $0(0 \%)$ & \\
\hline
\end{tabular}

IQR, Interquartile range.

violations (did not receive study medication per protocol), leaving 50 patients for analysis. In total, 34 remained in the aspirin arms and 16 in the clopidogrel arm (Figure 1).

The patients were well matched for baseline characteristics, in particular for the use of atorvastatin (Table 1). For patients randomly assigned to receive clopidogrel, the mean \pm SD aggregation with $1.1 \mu \mathrm{g} / \mathrm{L}$ collagen as an agonist on day 5 was $99 \% \pm 15 \%$ relative to baseline. At the same time, the $\mathrm{EC}_{50}$ concentrations were not raised versus baseline for the entire panel of agonists $(-0.04 \mu \mathrm{g} / \mathrm{L}$ for collagen, $-0.01 \mu \mathrm{mol} / \mathrm{L}$ for epinephrine, and $-0.02 \mu \mathrm{mol} / \mathrm{L}$ for ADP).

For aspirin, the mean aggregation with $1.1 \mu \mathrm{g} / \mathrm{L}$ collagen as an agonist was $56 \% \pm 15 \%$ relative to baseline on day 5 . The mean difference in aggregation between the two groups at this time point was $42 \%$ (95\% confidence interval $27 \%$ $56 \%$ in favor of aspirin. Further results in both treatment arms are presented in Table 2.

\section{Discussion}

There is no stimuli as potent as cardiopulmonary bypass for provoking an inflammatory response. The magnitude of the surgery and exposure to the extracorporeal circuit result in such marked platelet activation that normal pharmacologic response to antiplatelet agents cannot be assumed under these conditions. In healthy volunteers, inhibition of platelet aggregation by clopidogrel occurs 1 hour after dosing, with a mean percentage inhibition of $48.1 \%$ by day 5 with 5 $\mu \mathrm{mol} / \mathrm{L}$ ADP. ${ }^{5}$ However, we found no evidence of inhibition of platelet aggregation by clopidogrel with either ADP, collagen, or epinephrine by day 5 after coronary surgery.

With respect to a recent suggestion regarding the interaction between atorvastatin and clopidogrel ${ }^{6}$, we report that the absence of platelet inhibition was observed uniformly in all patients in the clopidogrel arm regardless of whether they were receiving atorvastatin, simvastatin, or pravastatin.

Unfortunately, our study was on the comparative efficacies of three antiplatelet regimens in the early postoperative period and was not focused on why clopidogrel would be ineffective, which was an unexpected finding on interim analysis. Although we assume that the inflammatory response generated from cardiopulmonary bypass is important, it is impossible to discern whether our findings could be explained by inadequate clopidogrel dosage under adverse conditions, high platelet turnover, platelet receptor dysregulation, or the effects of trauma independent of cardiopulmonary bypass. The most likely explanation is an interaction of factors, and clearly other studies are required to provide further insight to the weight of each individual contribution or to offer alternative explanation.

\section{Clinical Implications}

What does matter is the timing of antiplatelet administration that is critical within the first week after surgery for the effects of improved graft patency and survival. ${ }^{7}$ An early trial revealed that the frequency of new graft occlusions was not improved by aspirin after the first 9 days. ${ }^{8}$ More precisely, we assume that the effects of an antiplatelet drug need to be evident within this time (if it is the antiplatelet action that is important in the preservation of graft patency). If $75 \mathrm{mg}$ clopidogrel were to be used as the sole antiplatelet agent, this would be akin to giving patients a placebo in the first week, as previous work suggested that the antiplatelet effects of clopidogrel commences between the ninth and 28th postoperative days. ${ }^{9}$

Although the risk of recurrent ischemic events may be reduced by clopidogrel administration in patients with previous cardiac surgery, it is important to note that clopidogrel was not started immediately after surgery in the CAPRIE study, and patients could have received aspirin right up to entry into the trial. The results of our trial cannot be extrapolated beyond the early postoperative period, and we do not suggest that clopidogrel is ineffective in the long term. However, neither can the beneficial results of clopidogrel (as reported by CAPRIE) be safely extrapolated to the early postoperative phase. We have demonstrated the inefficacy of clopidogrel in this critical time frame. In view of the interim data, the clopidogrel arm has been withdrawn from our trial as we continue to evaluate the efficacy of lowversus medium-dose aspirin.

\section{Conclusion}

Because of its inability to inhibit platelet aggregation, clopidogrel at a dose of $75 \mathrm{mg}$ or less should not be used as the sole antiplatelet agent early after coronary artery bypass surgery. 


\begin{tabular}{|c|c|c|c|}
\hline & Aspirin & Clopidogrel & $P$ value \\
\hline \multicolumn{4}{|c|}{ Percentage aggregation (day 5 as percentage of baseline) } \\
\hline Collagen $1.1 \mu \mathrm{g} / \mathrm{L}$ (mean $\pm \mathrm{SD}$ ) & $57 \pm 26$ & $99 \pm 15$ & $<.001$ \\
\hline $\mathrm{ADP} \mu \mathrm{mol} / \mathrm{L}(\mathrm{mean} \pm \mathrm{SD})$ & $115 \pm 83$ & $111 \pm 55$ & .87 \\
\hline Epinephrine $0.5 \mu \mathrm{mol} / \mathrm{L}$ (median, IQR) & 53 (31 to 78 ) & 99 (85 to 116$)$ & .027 \\
\hline \multicolumn{4}{|l|}{ Difference in $\mathrm{EC}_{50}$ (day 5 minus baseline) } \\
\hline Collagen ( $\mu \mathrm{g} / \mathrm{L}$, median, IQR) & $0.92(0.71$ to 1.4$)$ & $-0.04(-0.10$ to 0.050$)$ & $<.001$ \\
\hline ADP ( $\mu \mathrm{mol} / \mathrm{L}$, median, IQR) & $0.17(-0.17$ to 0.46$)$ & $-0.019(-0.46$ to 0.12$)$ & .23 \\
\hline Epinephrine ( $\mu \mathrm{mol} / \mathrm{L}$, median, IQR) & $1.3(0.25$ to 3.9$)$ & $-0.013(-0.29$ to 0.088$)$ & .001 \\
\hline
\end{tabular}

$I Q R$, Interquartile range; $E C_{50}$, effective concentration of agonist to induce $50 \%$ aggregation.

We gratefully acknowledge Jillian Redpath, Emma Kadri, and Elizabeth Bligh from the Papworth Hospital Pharmacy for their assistance, Kate Sheridan for database management and laboratory analysis, Andrew Trull for project team assistance, Papworth Hospital Research and Development Department for project management, and the nurses of the Surgical Unit in Papworth Hospital.

\section{References}

1. Lim E, Ali Z, Ali A, Routledge T, Edmonds L, Altman DG, et al. Indirect comparison meta-analysis of aspirin therapy after coronary surgery. BMJ. 2003;327:1309.

2. Zimmermann N, Kienzle P, Weber AA, Winter J, Gams E, Schror K, et al. Aspirin resistance after coronary artery bypass grafting. $J$ Thorac Cardiovasc Surg. 2001;121:982-4.

3. Bhatt DL, Chew DP, Hirsch AT, Ringleb PA, Hacke W, Topol EJ. Superiority of clopidogrel versus aspirin in patients with prior cardiac surgery. Circulation. 2001;103:363-8.
4. Born GV. Aggregation of blood platelets by adenosine diphosphate and its reversal. Nature. 1962;164:927-9.

5. Savcic M, Hauert J, Bachmann F, Wyld PJ, Geudelin B, Cariou R. Clopidogrel loading dose regimens: kinetic profile of pharmacodynamic response in healthy subjects. Semin Thromb Hemost. 1999;25(Suppl 2):15-9.

6. Lau WC, Waskell LA, Watkins PB, Neer CJ, Horowitz K, Hopp AS, et al. Atorvastatin reduces the ability of clopidogrel to inhibit platelet aggregation: a new drug-drug interaction. Circulation. 2003;107: 32-7.

7. Mangano DT. Aspirin and mortality from coronary bypass surgery. $N$ Engl J Med. 2002;347:1309-17.

8. Goldman S, Copeland J, Moritz T, Henderson W, Zadina K, Ovitt T, et al. Saphenous vein graft patency 1 year after coronary artery bypass surgery and effects of antiplatelet therapy. Results of a Veterans Administration Cooperative Study. Circulation. 1989;80:1190-7.

9. David JL, Limet R. Antiplatelet activity of clopidogrel in coronary artery bypass graft surgery patients. Thromb Haemost. 1999;82:141721. 